\title{
Influence of INM on Vegetative Growth, Fruiting, Yield and Soil Physical Characters in Papaya (Carica papaya L.)
}

\author{
Amar Singh $^{*}$ and V. K. Tripathi \\ Department of Horticulture, C. S. Azad University of Agriculture and Technology, \\ Kanpur-208 002 (U.P.), India \\ *Corresponding author
}

\begin{tabular}{l} 
K e y w o r d s \\
$\begin{array}{l}\text { Papaya, Integrated } \\
\text { Nutrient } \\
\text { Management, } \\
\text { Growth, Fruiting, } \\
\text { Yield and Soil } \\
\text { physical characters }\end{array}$ \\
\hline Article Info \\
\hline $\begin{array}{l}\text { Accepted: } \\
\text { 25 September } 2020 \\
\text { Available Online: } \\
\text { 10 October } 2020\end{array}$ \\
\hline
\end{tabular}

\section{A B S T R A C T}

An experiment was carried out in the Department of Horticulture, Chandra Shekhar Azad University of Agriculture and Technology, Kanpur (U.P.), India, during 2015-16 and 2016-17 to study the influence of integrated nutrient management on vegetative growth, fruiting, yield and soil physical characters in papaya (Carica papaya L.). For this plant of Sapna cultivar was planted on $20^{\text {th }}$ March during both years of experimentation at a spacing of $2.0 \times 2.0 \mathrm{~m}$. There were eighteen treatments comprising Azotobacter, PSB and vermicompost with graded dose of RDF including one control, replicated thrice in randomized block design. All treatments were applied at the time of planting in the field. The data of both the years of experiment were analyzed which clearly shows that maximum plant spread from North to South direction and from East to West direction, maximum number of leaves and font area, biomass per plant, number of flowers, number of fruits set and yield per plant with minimum fruiting height and fruit developmental period were recorded with the application of RDF 75\% + Azotobacter $100 \mathrm{~g}+$ PSB $100 \mathrm{~g}$ + vermicompost $2 \mathrm{~kg} /$ plant, which was significantly higher than the control, whereas the minimum plant spread from North to South direction and from East to West direction, minimum number of leaves and font area, biomass per plant, number of flowers, number of fruits set and yield per plant with maximum fruiting height and fruit developmental period were recorded under unfertilized plants during both years of experimentation. As various soil physical characters are concerned maximum available potassium, organic carbon per cent, available nitrogen, available phosphorus with minimum soil $\mathrm{pH}$ and soil electrical conductivity were recorded in the field fertilized with RDF 75\%+ Azotobacter $100 \mathrm{~g}+$ PSB $100 \mathrm{~g}+$ vermicompost $2 \mathrm{~kg} /$ plant, whereas the minimum available potassium, available nitrogen, available phosphorus, minimum organic carbon per cent with maximum soil $\mathrm{pH}$ and electrical conductivity were recorded in field kept under control, where non amount of fertilizers were applied during both years of present investigation.

\section{Introduction}

Papaya (Carica papaya L.), a member of family Caricaceae, is a fast growing, 1 to 5 meter in height, perennial plant, which is basically a tropical fruit plant and commercially grown in tropical and subtropical areas. The optimum temperature for 
papaya is reported to be $21^{\circ}$ to $33^{\circ} \mathrm{C}$. In India, it is being cultivated in an area of $1.38 \mathrm{Lakh}$ ha with a total production of 5.99 Lakh MT and average productivity is 43.27 MT per hectare (Anonymous (2018). Papaya is a cheap and rich source of vitamins and minerals in the daily diet of millions of people. The latex obtained from raw papaya fruit is used for the preparation of an enzyme papain, which helps in the digestion of proteins, because of its proteolytic enzymatic activity. In addition to these unripe fruits are also used as vegetables, whereas the ripe fruits are very delicious in taste, which are used as dessert fruit. Its higher nutritive value, huge industrial importance, high remunerative price and fruiting throughout the year, put the papaya fruits to an important place in fruit industry and make this an important fruit for the growers, consumers, traders, exporter and processors.

An INM (Integrated Nutrient Management) is one of the most effective alternatives which involve combined use of chemical fertilizers, organic manures and bio-fertilizers for the maintenance of long-term soil fertility and productivity along with sustainable crop production. Organic manures mostly enhance the nutrient availability in order to improve the soil structure, texture, tilth and better environment for root development and aeration. Bio-fertilizers like Azotobacter and Phosphate Solubilising Bacteria (PSB) results an increased availability of nitrogen and phosphorus nutrients in the soil. Contrary to various chemical fertilizers, organic manures and bio-fertilizers are available indigenously at lower cost which also improve soil health and enhanced crop yield per unit of applied nutrient and there by save energy, keeping in all above facts in view, the present investigation was carried out to standardized most suitable combination of Azotobacter, PSB and vermicompost with a dose of chemical fertilizers in an integrated way to get increased vegetative growth, fruiting and higher yield of papaya (Carica papaya L.) fruits with improved soil physical characters.

\section{Materials and Methods}

The present investigation was carried out in the garden, Department of Horticulture, C.S. Azad University of Agriculture \& Technology Kanpur during two subsequent years i.e., 2015-16 and 2016-17. The experiments were laid out in Randomized Block Design with eighteen treatments viz., $\mathrm{T}_{0}$ (No amount of fertilizers), $T_{1}$ (recommended dose of fertilizers (RDF)-200:200:300 g/plant), $\mathrm{T}_{2}$ (RDF 75\%+ Azotobacter $50 \mathrm{~g}+$ PSB 50 g/plant), $\mathrm{T}_{3}$ (RDF $75 \%+$ Azotobacter $50 \mathrm{~g}+$ PSB $50 \mathrm{~g}+$ vermicompost $1 \mathrm{~kg} /$ plant), $\mathrm{T}_{4}$ (RDF 75\%+ Azotobacter $50 \mathrm{~g}+$ PSB $50 \mathrm{~g}+$ vermicompost $1.5 \mathrm{~kg} /$ plant), $\mathrm{T}_{5}(\mathrm{RDF} 75 \%+$ Azotobacter $50 \mathrm{~g}+$ PSB $50 \mathrm{~g}+$ vermicompost $2 \mathrm{~kg} / \mathrm{plant}), \mathrm{T}_{6}(\mathrm{RDF} 75 \%+$ Azotobacter 100 g+ PSB 100 g/plant), $\mathrm{T}_{7}$ (RDF 75\%+ Azotobacter $100 \mathrm{~g}+$ PSB $100 \mathrm{~g}+$ vermicompost $1 \mathrm{~kg} /$ plant), $\mathrm{T}_{8}$ (RDF $75 \%+$ Azotobacter $100 \mathrm{~g}+$ PSB $100 \mathrm{~g}+$ vermicompost $1.5 \mathrm{~kg} /$ plant), $\mathrm{T}_{9}$ (RDF $75 \%+$ Azotobacter $100 \mathrm{~g}+$ PSB $100 \mathrm{~g}+$ vermicompost $2 \mathrm{~kg} /$ plant), $\mathrm{T}_{10}(\mathrm{RDF} 50 \%+$ Azotobacter $50 \mathrm{~g}+$ PSB $50 \mathrm{~g} / \mathrm{plant}), \mathrm{T}_{11}$ (RDF $50 \%+$ Azotobacter $50 \mathrm{~g}+$ PSB $50 \mathrm{~g}+$ vermicompost $1 \mathrm{~kg} /$ plant), $\mathrm{T}_{12}(\mathrm{RDF} 50 \%+$ Azotobacter $50 \mathrm{~g}+$ PSB $50 \mathrm{~g}+$ vermicompost $1.5 \mathrm{~kg} / \mathrm{plant}$ ), $\mathrm{T}_{13}$ (RDF $50 \%+$ Azotobacter 50 $\mathrm{g}+$ PSB $50 \mathrm{~g}+$ vermicompost $2 \mathrm{~kg} /$ plant), $\mathrm{T}_{14}$ (RDF 50\%+ Azotobacter $100 \mathrm{~g}+$ PSB 100 g/plant), $\mathrm{T}_{15}$ (RDF 50\%+ Azotobacter $100 \mathrm{~g}+$ PSB $100 \mathrm{~g}+$ vermicompost $1 \mathrm{~kg} /$ plant), $\mathrm{T}_{16}$ (RDF 50\%+ Azotobacter $100 \mathrm{~g}+$ PSB $100 \mathrm{~g}$ + vermicompost $1.5 \mathrm{~kg} / \mathrm{plant}$ ), $\mathrm{T}_{17}$ (RDF $50 \%+$ Azotobacter $100 \mathrm{~g}+$ PSB $100 \mathrm{~g}+$ vermicompost $2 \mathrm{~kg} /$ plant). Planting was done at a distance of $2 \mathrm{~m} \times 2 \mathrm{~m}$ on $20^{\text {th }}$ March during both years of experimentation i.e., 2015-16 and 2016-17, using 'Sapna' cultivar. Two plants are used as a unit. 
Observations on plants spread from North to South and East to West direction were measured by measuring tape along with measuring pole in centimeter, whereas number of leaves per plant was counted started from the time of transplanting and to the harvesting of last fruit. Font of leaves was measured by using leaf area meter and biomass production of plant was recorded by weighing the whole plant, including shoot and leaves expressed in kilograms $(\mathrm{kg})$. Data on number of flowers and fruits set per plant were recorded by counting at ten days interval during entire fruiting season. Height at which first fruit appeared was recorded from the base of the plant to denote the fruiting height, whereas the period between fruit set and fruit maturity was calculated as fruits developmental period. At the time of harvesting, fruit were weighted to calculate yield per plant. As soil physical characters are concerned, soil $\mathrm{pH}$ was analyzed with the help of digital $\mathrm{pH}$ meter, Electrical conductivity by digital conductivity meter as advocated by Jackson, (1973). Organic carbon (\%) by rapid titration method as describe by Walkley and Black (1934), available nitrogen by alkaline potassium permanganate method as suggested by Subbiah and Asija (1956), available phosphorus $\left(\mathrm{kg} \mathrm{ha}^{-1}\right)$ by Olsen's method as described by Olsen et al., (1954) and available potassium $\left(\mathrm{kg} \mathrm{ha}^{-1}\right)$ by flame photometer with the use of saturation extract of soil as describe by Jackson (1973).

\section{Results and Discussion}

\section{Plant spread (North to South and East to West direction)}

Plant spread in all direction was significantly more over control when Azotobacter, PSB and vermicompost were used in combinations with different doses of RDF during both years of experimentation (Table 1). Maximum plant spread from North to South direction (191.08 and $189.15 \mathrm{~cm}$, respectively) and from East to West direction (182.22 and $173.86 \mathrm{~cm}$, respectively) were recorded with the application of RDF $75 \%+$ Azotobacter $100 \mathrm{~g}$ + PSB $100 \mathrm{~g}+$ vermicompost $2 \mathrm{~kg} /$ plant, which was significantly higher than the control, whereas the minimum plant spread from North to South direction (140.85 and $139.26 \mathrm{~cm}$, respectively) and from East to West direction (129.15 and $126.89 \mathrm{~cm}$, respectively) was recorded under unfertilized plants during both years of experimentation. The marked increase in plant spread in all direction with the use of bio-fertilizers and vermicompost along with graded dose of NPK may be due to the fact that absorbed nutrients combined with carbohydrates in leaves could lead to the formation of amino acids, nucleic acid, proteins, chlorophyll, alkaloid and amides. These complex compounds are responsible for building up of new tissues and are associated in a number of metabolic steps in fruit crops. Bio-fertilizers and vermicompost are also known to enrich the soil by biological N-fixation and improving the availability of different nutrients to plants, which results more vegetative growth in all directions. These results are in conformity with the findings of Kirankumar et al., (2017) in guava, Gautam et al., (2012) in Mango and Srivastava et al., (2014) in papaya.

\section{Fruiting height}

Data presented in Table 1 clearly shows that during both years of experimentation fruiting height of plants were significantly decreased over control when Azotobacter, PSB and vermicompost were used in combinations with different doses of RDF. Plants having minimum fruiting height (36.24 and 35.92 $\mathrm{cm}$, respectively) were recorded when they were fertilized with RDF $75 \%+$ Azotobacter $100 \mathrm{~g}+$ PSB $100 \mathrm{~g}+$ vermicompost 2 $\mathrm{kg} /$ plant $\left(\mathrm{T}_{9}\right)$ and this was significantly higher 
than rest all other treatments except $\mathrm{T}_{8}, \mathrm{~T}_{1}$, $\mathrm{T}_{15}, \mathrm{~T}_{16}, \mathrm{~T}_{17}, \mathrm{~T}_{6}$ and $\mathrm{T}_{7}$. On the contrary, maximum fruiting height (46.12 and 45.96 $\mathrm{cm}$, respectively) was recorded in untreated control plants during both years of experimentation. The results with respect to fruiting height is also in conformity with the findings of Srivastava (2008), who reported that $100 \%$ NPK + FYM + Azotobacter + PSB showed significant differences as compared with the control (100\% NPK + FYM) for measured fruiting height in papaya.

\section{Number of leaves and font area}

During both years of investigation, the number of leaves and font area were significantly increased over control when Azotobacter, PSB and vermicompost were used in combinations with different doses of RDF (Table-1). Plants fertilized with RDF $75 \%$ + Azotobacter $100 \mathrm{~g}+$ PSB $100 \mathrm{~g}+$ vermicompost $2 \mathrm{~kg} /$ plant produced significantly maximum number of leaves (35.54 and 36.52, respectively) and maximum font area $(33.19$ and $33.20 \mathrm{~cm})$, whereas the minimum number of leaves (26.00 and 25.65, respectively) and font area (21.51 and 20.75 $\mathrm{cm}$, respectively) were recorded under untreated control plants during both years of experimentation. This increase in leaves number and font area during entire experimental period might be due to the continuous supply of available nutrient from organic and inorganic form and effect of bioactive substance produced by the application of bio-fertilizers.

Organic manures (vermicompost) along with bio-fertilizers also improve the aeration in the soil which ultimately improved the physiological activities inside the plant like plant height, stem girth, number of leaves and font area. The present results are in accordance to the findings of Srinu et al., (2017), Srivastava et al., (2014) in papaya,
Gupta and Tripathi (2012), Tripathi et al., (2015b) in strawberry cv. Chandler and Tripathi et al., (2013) in Isabgol.

\section{Biomass production (green weight)}

During both years of experimentation biomass production (green weight) was significantly more over control when Azotobacter, PSB and vermicompost were used in combinations with different doses of RDF (Table 1). When the plants were fertilized with RDF $75 \%+$ Azotobacter $100 \mathrm{~g}+$ PSB $100 \mathrm{~g}+$ vermicompost $2 \mathrm{~kg} /$ plant produced maximum biomass (29.00 and $28.34 \mathrm{~kg}$, respectively) per plant followed by RDF 75\%+Azotobacter $100 \mathrm{~g}$ + PSB $100 \mathrm{~g}+$ vermicompost 1.5 $\mathrm{kg} /$ plant fertilized plants which produces 28.00 and $27.37 \mathrm{~kg}$, respectively biomass per plant on green weight. On the contrary, minimum biomass $(12.00$ and $11.65 \mathrm{~kg}$, respectively) per plant on green weight was produced under untreated control plants in which non amount of fertilizers were applied during both years of experimentation.

Plants treated with Azotobacter, PSB and vermicompost showed significantly higher shoot biomass accumulation over control and untreated ones might be due to the induction of growth hormones, which stimulated cell division, cell elongation, activate the photosynthesis process. Further application of organic manure, there was also significant increase in shoot biomass content over control.

These results are corroborated with the findings of Sukhada et al., (1995) who reported that papaya plants inoculated with $G$. mosseae exhibited an increased dry matter (26.6\%) under sterile conditions. The present results are in accordance to the findings of Kirankumar et al., (2017) in guava and Mamta et al., (2017) in papaya. 
Table.1 Influence of Integrated Nutrient Management on growth parameters of papaya (Carica papaya L.)

\begin{tabular}{|c|c|c|c|c|c|c|c|c|c|c|}
\hline \multirow[t]{2}{*}{ Treatments } & \multicolumn{2}{|c|}{$\begin{array}{l}\text { Plant spread (North- } \\
\text { South) }(\mathrm{cm})\end{array}$} & \multicolumn{2}{|c|}{$\begin{array}{l}\text { Plant spread (East-West) } \\
(\text { (cm) }\end{array}$} & \multicolumn{2}{|c|}{$\begin{array}{c}\text { Number of leaves } \\
\text { per plant }\end{array}$} & \multicolumn{2}{|c|}{ Font of leaf $(\mathrm{cm})$} & \multicolumn{2}{|c|}{$\begin{array}{c}\text { Biomass production of } \\
\text { plant (green weight in } \mathrm{kg} \text { ) }\end{array}$} \\
\hline & 2015-16 & 2016-17 & 2015-16 & 2016-17 & 2015-16 & 2016-17 & 2015-16 & 2016-17 & 2015-16 & 2016-17 \\
\hline $\mathbf{T}_{\mathbf{0}}$ & 140.85 & 139.26 & 129.15 & 126.89 & 26.00 & 25.65 & 21.51 & 20.75 & 12.00 & 11.65 \\
\hline $\mathbf{T}_{1}$ & 184.20 & 180.12 & 166.06 & 158.50 & 33.33 & 31.45 & 31.30 & 31.34 & 27.00 & 26.39 \\
\hline $\mathbf{T}_{2}$ & 153.84 & 151.28 & 142.88 & 136.44 & 28.76 & 27.87 & 27.16 & 26.99 & 18.46 & 18.08 \\
\hline $\mathbf{T}_{\mathbf{3}}$ & 159.01 & 157.08 & 145.56 & 138.98 & 26.57 & 27.24 & 28.79 & 28.96 & 19.67 & 19.25 \\
\hline $\mathbf{T}_{4}$ & 162.65 & 163.42 & 146.68 & 140.05 & 33.24 & 31.67 & 30.18 & 30.20 & 20.00 & 19.58 \\
\hline $\mathbf{T}_{5}$ & 170.86 & 169.95 & 163.11 & 155.68 & 34.24 & 32.62 & 30.93 & 30.82 & 22.00 & 21.52 \\
\hline $\mathbf{T}_{6}$ & 175.86 & 171.29 & 149.28 & 142.53 & 29.97 & 29.86 & 31.00 & 31.05 & 20.00 & 19.58 \\
\hline $\mathbf{T}_{7}$ & 180.23 & 175.58 & 156.05 & 148.97 & 30.91 & 30.76 & 31.80 & 31.78 & 17.33 & 16.98 \\
\hline $\mathbf{T}_{8}$ & 187.64 & 184.64 & 176.07 & 168.02 & 33.44 & 33.49 & 32.89 & 32.85 & 28.00 & 27.37 \\
\hline $\mathbf{T}_{9}$ & 191.08 & 189.15 & 182.22 & 173.86 & 35.54 & 36.52 & 33.19 & 33.20 & 29.00 & 28.34 \\
\hline $\mathbf{T}_{10}$ & 152.61 & 150.20 & 150.52 & 143.71 & 28.33 & 27.45 & 23.65 & 26.60 & 17.45 & 17.09 \\
\hline $\mathbf{T}_{11}$ & 159.25 & 155.45 & 157.73 & 150.56 & 27.79 & 26.50 & 24.49 & 24.45 & 22.00 & 21.52 \\
\hline $\mathbf{T}_{12}$ & 163.09 & 160.91 & 154.06 & 147.07 & 31.33 & 30.86 & 25.84 & 25.86 & 19.00 & 18.60 \\
\hline $\mathbf{T}_{13}$ & 165.27 & 164.32 & 152.64 & 145.72 & 30.33 & 31.81 & 26.66 & 26.38 & 23.00 & 22.50 \\
\hline $\mathbf{T}_{14}$ & 174.21 & 171.84 & 147.07 & 140.42 & 27.24 & 28.97 & 27.39 & 27.38 & 15.67 & 15.36 \\
\hline $\mathbf{T}_{15}$ & 178.40 & 177.24 & 151.90 & 145.02 & 29.33 & 27.96 & 29.22 & 29.24 & 18.00 & 17.63 \\
\hline $\mathbf{T}_{16}$ & 181.51 & 180.10 & 161.23 & 153.89 & 32.33 & 30.81 & 29.81 & 29.83 & 21.00 & 20.55 \\
\hline $\mathbf{T}_{17}$ & 185.07 & 184.80 & 177.50 & 169.37 & 33.15 & 32.98 & 30.10 & 30.14 & 26.00 & 27.44 \\
\hline SEm \pm & 9.29 & 9.01 & 8.11 & 8.75 & 1.76 & 2.00 & 2.07 & 2.28 & 1.95 & 1.98 \\
\hline CD $_{5 \%}$ level & 28.17 & 27.34 & 24.59 & 26.55 & 5.34 & 6.08 & 6.29 & 6.92 & 5.91 & 5.99 \\
\hline CV & 9.44 & 9.28 & 8.99 & 10.16 & 9.95 & 11.47 & 12.52 & 13.74 & 16.23 & 16.62 \\
\hline
\end{tabular}

Treatments notations: $\mathrm{T}_{0}$ (No amount of fertilizers), $\mathrm{T}_{1}$ (recommended dose of fertilizers (RDF)-200:200:300 g/plant), $\mathrm{T}_{2}$ (RDF 75\% + Azotobacter 50 g + PSB 50 $\mathrm{g} / \mathrm{plant}), \mathrm{T}_{3}$ (RDF 75\%+ Azotobacter $50 \mathrm{~g}+\mathrm{PSB} 50 \mathrm{~g}+$ vermicompost $\left.1 \mathrm{~kg} / \mathrm{plant}\right), \mathrm{T}_{4}(\mathrm{RDF} 75 \%+$ Azotobacter $50 \mathrm{~g}+\mathrm{PSB} 50 \mathrm{~g}+\mathrm{vermicompost} 1.5 \mathrm{~kg} / \mathrm{plant}), \mathrm{T}_{5}$

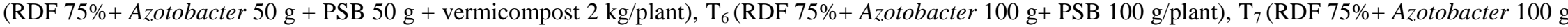
+ PSB $100 \mathrm{~g}+$ vermicompost $1 \mathrm{~kg} / \mathrm{plant}), \mathrm{T}_{8}(\mathrm{RDF} 75 \%+$ Azotobacter $100 \mathrm{~g}+\mathrm{PSB} 100 \mathrm{~g}+$ vermicompost $1.5 \mathrm{~kg} / \mathrm{plant}), \mathrm{T}_{9}(\mathrm{RDF} 75 \%+$ Azotobacter $100 \mathrm{~g}+$ PSB $100 \mathrm{~g}+$ vermicompost $2 \mathrm{~kg} / \mathrm{plant}), \mathrm{T}_{10}(\mathrm{RDF} 50 \%+$ Azotobacter $50 \mathrm{~g}+$ PSB $50 \mathrm{~g} / \mathrm{plant}), \mathrm{T}_{11}$ (RDF $50 \%+$ Azotobacter $50 \mathrm{~g}+\mathrm{PSB} 50 \mathrm{~g}+\mathrm{vermicompost} 1$ $\mathrm{kg} / \mathrm{plant}), \mathrm{T}_{12}(\mathrm{RDF} 50 \%+$ Azotobacter $50 \mathrm{~g}+$ PSB $50 \mathrm{~g}+$ vermicompost $1.5 \mathrm{~kg} / \mathrm{plant}), \mathrm{T}_{13}$ (RDF $50 \%+$ Azotobacter $50 \mathrm{~g}+$ PSB $50 \mathrm{~g}+\mathrm{vermicompost} 2$ $\mathrm{kg} / \mathrm{plant}), \mathrm{T}_{14}$ (RDF 50\%+ Azotobacter $\left.100 \mathrm{~g}+\mathrm{PSB} 100 \mathrm{~g} / \mathrm{plant}\right), \mathrm{T}_{15}$ (RDF 50\%+ Azotobacter $\left.100 \mathrm{~g}+\mathrm{PSB} 100 \mathrm{~g}+\mathrm{vermicompost} 1 \mathrm{~kg} / \mathrm{plant}\right), \mathrm{T}_{16}(\mathrm{RDF} 50 \%+$ Azotobacter $100 \mathrm{~g}+$ PSB $100 \mathrm{~g}+$ vermicompost $1.5 \mathrm{~kg} / \mathrm{plant}), \mathrm{T}_{17}$ (RDF 50\%+ Azotobacter $\left.100 \mathrm{~g}+\mathrm{PSB} 100 \mathrm{~g}+\mathrm{vermicompost} 2 \mathrm{~kg} / \mathrm{plant}\right)$ 
Table2 Influence of Integrated Nutrient Management on fruiting and yield parameters of papaya (Carica papaya L.)

\begin{tabular}{|c|c|c|c|c|c|c|c|c|}
\hline \multirow[t]{2}{*}{ Treatments } & \multicolumn{2}{|c|}{$\begin{array}{l}\text { Fruit developmental period } \\
\text { (Days) }\end{array}$} & \multicolumn{2}{|c|}{ Number of flowers per plant } & \multicolumn{2}{|c|}{$\begin{array}{c}\text { Number of fruits set per } \\
\text { plant }\end{array}$} & \multicolumn{2}{|c|}{ Fruit yield (kg/tree) } \\
\hline & 2015-16 & 2016-17 & $2015-16$ & 2016-17 & 2015-16 & 2016-17 & $2015-16$ & 2016-17 \\
\hline $\mathbf{T}_{\mathbf{0}}$ & 164.00 & 163.26 & 73.86 & 72.24 & 24.00 & 22.80 & 22.34 & 23.49 \\
\hline $\mathbf{T}_{1}$ & 144.23 & 142.20 & 103.85 & 103.28 & 40.00 & 38.00 & 58.80 & 52.98 \\
\hline $\mathbf{T}_{2}$ & 159.37 & 158.33 & 77.00 & 75.15 & 28.00 & 27.56 & 29.38 & 26.47 \\
\hline $\mathbf{T}_{3}$ & 152.19 & 151.34 & 84.76 & 80.25 & 30.00 & 28.50 & 35.70 & 36.17 \\
\hline $\mathbf{T}_{4}$ & 160.00 & 158.08 & 89.00 & 84.55 & 32.00 & 30.40 & 39.81 & 35.87 \\
\hline $\mathbf{T}_{5}$ & 155.67 & 154.68 & 97.25 & 94.05 & 36.00 & 34.20 & 49.18 & 44.31 \\
\hline $\mathbf{T}_{6}$ & 159.93 & 155.86 & 85.10 & 83.95 & 28.50 & 27.70 & 31.20 & 28.11 \\
\hline $\mathbf{T}_{7}$ & 159.33 & 154.96 & 91.00 & 89.45 & 31.00 & 29.45 & 38.22 & 34.44 \\
\hline $\mathbf{T}_{8}$ & 141.32 & 141.65 & 104.20 & 104.75 & 41.50 & 39.43 & 62.94 & 56.71 \\
\hline $\mathbf{T}_{9}$ & 140.25 & 141.37 & 104.69 & 104.80 & 43.00 & 40.85 & 67.08 & 60.44 \\
\hline $\mathbf{T}_{10}$ & 155.34 & 151.67 & 79.00 & 75.05 & 25.18 & 24.97 & 26.93 & 26.26 \\
\hline $\mathbf{T}_{11}$ & 154.35 & 153.42 & 81.30 & 80.15 & 29.00 & 27.55 & 32.66 & 31.83 \\
\hline $\mathbf{T}_{12}$ & 152.67 & 153.32 & 82.00 & 83.08 & 27.00 & 25.65 & 35.07 & 33.60 \\
\hline $\mathbf{T}_{13}$ & 155.00 & 144.35 & 84.22 & 82.50 & 28.00 & 26.60 & 35.17 & 32.69 \\
\hline $\mathbf{T}_{14}$ & 149.28 & 147.93 & 81.10 & 82.05 & 27.00 & 25.65 & 28.51 & 25.69 \\
\hline $\mathbf{T}_{15}$ & 147.59 & 146.20 & 88.00 & 87.60 & 33.00 & 31.35 & 35.54 & 32.03 \\
\hline $\mathbf{T}_{16}$ & 162.47 & 157.19 & 94.00 & 89.30 & 36.00 & 34.20 & 46.80 & 42.17 \\
\hline $\mathbf{T}_{17}$ & 163.24 & 160.23 & 98.00 & 93.10 & 38.00 & 36.10 & 53.58 & 48.28 \\
\hline SEm \pm & 4.50 & 4.53 & 4.08 & 3.18 & 2.29 & 1.81 & 2.33 & 1.94 \\
\hline CD $_{5 \%}$ level & 13.67 & 13.74 & 12.37 & 9.66 & 6.95 & 5.50 & 7.06 & 5.87 \\
\hline CV & 5.06 & 5.16 & 7.95 & 6.35 & 12.37 & 10.26 & 9.96 & 8.99 \\
\hline
\end{tabular}

Treatments notations: $\mathrm{T}_{0}$ (No amount of fertilizers), $\mathrm{T}_{1}$ (recommended dose of fertilizers (RDF)-200:200:300 g/plant), $\mathrm{T}_{2}$ (RDF 75\%+ Azotobacter 50 g + PSB 50 $\mathrm{g} / \mathrm{plant}$ ), $\mathrm{T}_{3}$ (RDF 75\%+ Azotobacter $50 \mathrm{~g}+$ PSB $50 \mathrm{~g}+$ vermicompost $1 \mathrm{~kg} / \mathrm{plant}$ ), $\mathrm{T}_{4}(\mathrm{RDF} 75 \%+$ Azotobacter $50 \mathrm{~g}+\mathrm{PSB} 50 \mathrm{~g}+\mathrm{vermicompost} 1.5 \mathrm{~kg} / \mathrm{plant}), \mathrm{T}_{5}$ (RDF 75\% + Azotobacter $50 \mathrm{~g}+$ PSB $50 \mathrm{~g}+$ vermicompost $2 \mathrm{~kg} / \mathrm{plant}$ ), $\mathrm{T}_{6}$ (RDF 75\%+Azotobacter $\left.100 \mathrm{~g}+\mathrm{PSB} 100 \mathrm{~g} / \mathrm{plant}\right), \mathrm{T}_{7}$ (RDF $75 \%+$ Azotobacter $100 \mathrm{~g}$ + PSB $100 \mathrm{~g}+$ vermicompost $1 \mathrm{~kg} / \mathrm{plant}), \mathrm{T}_{8}(\mathrm{RDF} 75 \%+$ Azotobacter $100 \mathrm{~g}+\mathrm{PSB} 100 \mathrm{~g}+$ vermicompost $1.5 \mathrm{~kg} / \mathrm{plant}), \mathrm{T}_{9}(\mathrm{RDF} 75 \%+$ Azotobacter $100 \mathrm{~g}+$ PSB $100 \mathrm{~g}+$ vermicompost $2 \mathrm{~kg} / \mathrm{plant}), \mathrm{T}_{10}(\mathrm{RDF} 50 \%+$ Azotobacter $50 \mathrm{~g}+$ PSB $50 \mathrm{~g} / \mathrm{plant}), \mathrm{T}_{11}$ (RDF $50 \%+$ Azotobacter $50 \mathrm{~g}+$ PSB $50 \mathrm{~g}+\mathrm{vermicompost} 1$ $\mathrm{kg} / \mathrm{plant}), \mathrm{T}_{12}$ (RDF $50 \%+$ Azotobacter $50 \mathrm{~g}+$ PSB $50 \mathrm{~g}+$ vermicompost $1.5 \mathrm{~kg} / \mathrm{plant}$ ), $\mathrm{T}_{13}$ (RDF $50 \%+$ Azotobacter $50 \mathrm{~g}+$ PSB $50 \mathrm{~g}+\mathrm{vermicompost} 2$ $\mathrm{kg} / \mathrm{plant}$ ), $\mathrm{T}_{14}$ (RDF 50\%+ Azotobacter $\left.100 \mathrm{~g}+\mathrm{PSB} 100 \mathrm{~g} / \mathrm{plant}\right), \mathrm{T}_{15}$ (RDF 50\%+ Azotobacter $\left.100 \mathrm{~g}+\mathrm{PSB} 100 \mathrm{~g}+\mathrm{vermicompost} 1 \mathrm{~kg} / \mathrm{plant}\right), \mathrm{T}_{16}$ (RDF 50\%+ Azotobacter $100 \mathrm{~g}+$ PSB $100 \mathrm{~g}+$ vermicompost $1.5 \mathrm{~kg} / \mathrm{plant}), \mathrm{T}_{17}$ (RDF 50\%+ Azotobacter $\left.100 \mathrm{~g}+\mathrm{PSB} 100 \mathrm{~g}+\mathrm{vermicompost} 2 \mathrm{~kg} / \mathrm{plant}\right)$ 
Table.3 Influence of Integrated Nutrient Management on soil physical characters in papaya (Carica papaya L.) field

\begin{tabular}{|c|c|c|c|c|c|c|c|c|c|c|c|c|}
\hline \multirow[t]{2}{*}{ Treatments } & \multicolumn{2}{|c|}{ Soil pH } & \multicolumn{2}{|c|}{$\begin{array}{l}\text { Electrical conductivity } \\
\left(\mathbf{d S m}^{-1}\right)\end{array}$} & \multicolumn{2}{|c|}{ Organic carbon $(\%)$} & \multicolumn{2}{|c|}{$\begin{array}{c}\text { Available } \\
\text { nitrogen }\left(\mathrm{kg} \mathrm{ha}^{-1}\right)\end{array}$} & \multicolumn{2}{|c|}{$\begin{array}{c}\text { Available } \\
\text { Phosphorus }\left(\mathrm{kg} \mathrm{ha}^{-1}\right)\end{array}$} & \multicolumn{2}{|c|}{$\begin{array}{c}\text { Available potassium } \\
\qquad\left(\mathrm{kg} \mathrm{ha}^{-1}\right)\end{array}$} \\
\hline & $2015-16$ & $2016-17$ & $2015-16$ & 2016-17 & $2015-16$ & $2016-17$ & $2015-16$ & 2016-17 & $2015-16$ & 2016-17 & $2015-16$ & 2016-17 \\
\hline $\mathbf{T}_{0}$ & 7.65 & 7.70 & 0.43 & 0.44 & 0.33 & 0.34 & 251.26 & 252.34 & 20.35 & 21.95 & 260.17 & 261.24 \\
\hline $\mathbf{T}_{1}$ & 7.42 & 7.42 & 0.40 & 0.42 & 0.36 & 0.37 & 265.15 & 271.24 & 22.48 & 23.60 & 260.14 & 266.62 \\
\hline $\mathbf{T}_{2}$ & 7.40 & 7.38 & 0.39 & 0.38 & 0.38 & 0.39 & 265.14 & 264.19 & 23.73 & 24.30 & 265.80 & 266.29 \\
\hline $\mathbf{T}_{3}$ & 7.44 & 7.40 & 0.41 & 0.40 & 0.40 & 0.41 & 267.07 & 266.13 & 24.07 & 25.77 & 266.73 & 267.22 \\
\hline $\mathbf{T}_{4}$ & 7.46 & 7.41 & 0.42 & 0.41 & 0.41 & 0.42 & 268.84 & 268.91 & 24.94 & 26.08 & 267.50 & 269.00 \\
\hline $\mathbf{T}_{5}$ & 7.54 & 7.39 & 0.38 & 0.37 & 0.43 & 0.44 & 269.96 & 270.28 & 25.74 & 26.68 & 271.83 & 272.35 \\
\hline $\mathbf{T}_{6}$ & 7.44 & 7.39 & 0.40 & 0.39 & 0.39 & 0.40 & 265.48 & 268.53 & 23.48 & 24.65 & 266.14 & 267.63 \\
\hline $\mathbf{T}_{7}$ & 7.40 & 7.35 & 0.39 & 0.38 & 0.41 & 0.42 & 268.81 & 268.91 & 25.81 & 25.20 & 268.47 & 270.98 \\
\hline $\mathbf{T}_{8}$ & 7.37 & 7.32 & 0.36 & 0.35 & 0.42 & 0.43 & 270.14 & 270.65 & 27.14 & 26.95 & 269.82 & 271.31 \\
\hline $\mathbf{T}_{9}$ & 7.10 & 7.26 & 0.33 & 0.32 & 0.44 & 0.45 & 271.15 & 300.85 & 27.89 & 27.80 & 270.14 & 272.65 \\
\hline $\mathbf{T}_{10}$ & 7.43 & 7.35 & 0.39 & 0.38 & 0.37 & 0.38 & 264.92 & 262.97 & 23.14 & 24.12 & 265.58 & 266.07 \\
\hline $\mathbf{T}_{11}$ & 7.45 & 7.39 & 0.40 & 0.39 & 0.39 & 0.40 & 266.48 & 265.56 & 24.48 & 25.14 & 266.14 & 266.84 \\
\hline $\mathbf{T}_{12}$ & 7.44 & 7.37 & 0.38 & 0.39 & 0.40 & 0.41 & 268.01 & 267.41 & 24.40 & 25.81 & 267.00 & 268.50 \\
\hline $\mathbf{T}_{13}$ & 7.42 & 7.37 & 0.38 & 0.37 & 0.42 & 0.43 & 268.04 & 269.12 & 25.04 & 26.34 & 268.70 & 270.20 \\
\hline$T_{14}$ & 7.45 & 7.40 & 0.41 & 0.40 & 0.38 & 0.39 & 261.61 & 267.88 & 22.81 & 24.38 & 268.87 & 266.97 \\
\hline $\mathbf{T}_{15}$ & 7.41 & 7.36 & 0.37 & 0.36 & 0.39 & 0.40 & 266.34 & 269.40 & 24.34 & 24.73 & 268.00 & 270.18 \\
\hline $\mathbf{T}_{16}$ & 7.40 & 7.35 & 0.36 & 0.35 & 0.41 & 0.42 & 268.15 & 268.22 & 26.34 & 26.45 & 268.80 & 270.49 \\
\hline$T_{17}$ & 7.42 & 7.37 & 0.38 & 0.39 & 0.42 & 0.43 & 268.78 & 270.57 & 27.48 & 27.10 & 269.15 & 270.74 \\
\hline SEm \pm & 0.06 & 0.06 & 0.02 & 0.01 & 0.01 & 0.02 & 1.57 & 4.61 & 1.12 & 1.04 & 9.91 & 11.24 \\
\hline CD $_{5 \%}$ level & 0.20 & 0.17 & 0.05 & 0.04 & 0.04 & 0.05 & 4.77 & 13.99 & 3.39 & 3.15 & NS & NS \\
\hline CV & 1.51 & 1.33 & 7.44 & 5.34 & 5.77 & 6.93 & 1.02 & 2.97 & 7.84 & 7.09 & 6.43 & 7.25 \\
\hline
\end{tabular}

Treatments notations: $\mathrm{T}_{0}$ (No amount of fertilizers), $\mathrm{T}_{1}$ (recommended dose of fertilizers (RDF)-200:200:300 g/plant), $\mathrm{T}_{2}$ (RDF 75\% + Azotobacter 50 g + PSB 50 $\mathrm{g} / \mathrm{plant}), \mathrm{T}_{3}(\mathrm{RDF} 75 \%+$ Azotobacter $50 \mathrm{~g}+\mathrm{PSB} 50 \mathrm{~g}+$ vermicompost $1 \mathrm{~kg} / \mathrm{plant}), \mathrm{T}_{4}(\mathrm{RDF} 75 \%+$ Azotobacter $50 \mathrm{~g}+\mathrm{PSB} 50 \mathrm{~g}+\mathrm{vermicompost} 1.5 \mathrm{~kg} / \mathrm{plant}), \mathrm{T}_{5}$ (RDF 75\%+Azotobacter $50 \mathrm{~g}+$ PSB $50 \mathrm{~g}+$ vermicompost $2 \mathrm{~kg} / \mathrm{plant}), \mathrm{T}_{6}(\mathrm{RDF} 75 \%+$ Azotobacter $100 \mathrm{~g}+\mathrm{PSB} 100 \mathrm{~g} / \mathrm{plant}), \mathrm{T}_{7}(\mathrm{RDF} 75 \%+$ Azotobacter $100 \mathrm{~g}$ + PSB $100 \mathrm{~g}+$ vermicompost $1 \mathrm{~kg} / \mathrm{plant}), \mathrm{T}_{8}(\mathrm{RDF} 75 \%+$ Azotobacter $100 \mathrm{~g}+\mathrm{PSB} 100 \mathrm{~g}+$ vermicompost $1.5 \mathrm{~kg} / \mathrm{plant}), \mathrm{T}_{9}(\mathrm{RDF} 75 \%+$ Azotobacter $100 \mathrm{~g}+$ PSB $100 \mathrm{~g}+$ vermicompost $2 \mathrm{~kg} / \mathrm{plant}$ ), $\mathrm{T}_{10}$ (RDF $50 \%+$ Azotobacter $50 \mathrm{~g}+$ PSB $\left.50 \mathrm{~g} / \mathrm{plant}\right), \mathrm{T}_{11}$ (RDF 50\%+ Azotobacter $50 \mathrm{~g}+$ PSB $50 \mathrm{~g}+$ vermicompost 1 $\mathrm{kg} / \mathrm{plant}$ ), $\mathrm{T}_{12}$ (RDF $50 \%+$ Azotobacter $50 \mathrm{~g}+$ PSB $50 \mathrm{~g}+$ vermicompost $1.5 \mathrm{~kg} / \mathrm{plant}$ ), $\mathrm{T}_{13}$ (RDF $50 \%+$ Azotobacter $50 \mathrm{~g}+$ PSB $50 \mathrm{~g}+\mathrm{vermicompost} 2$ $\mathrm{kg} / \mathrm{plant}), \mathrm{T}_{14}(\mathrm{RDF} 50 \%+$ Azotobacter $100 \mathrm{~g}+$ PSB $100 \mathrm{~g} / \mathrm{plant}), \mathrm{T}_{15}(\mathrm{RDF} 50 \%+$ Azotobacter $100 \mathrm{~g}+$ PSB $100 \mathrm{~g}+$ vermicompost $1 \mathrm{~kg} / \mathrm{plant}), \mathrm{T}_{16}(\mathrm{RDF} 50 \%+$ Azotobacter $100 \mathrm{~g}+$ PSB $100 \mathrm{~g}+$ vermicompost $1.5 \mathrm{~kg} / \mathrm{plant}), \mathrm{T}_{17}(\mathrm{RDF} 50 \%+$ Azotobacter $100 \mathrm{~g}+$ PSB $100 \mathrm{~g}+$ vermicompost $2 \mathrm{~kg} / \mathrm{plant})$ 


\section{Fruit developmental period}

Fruit developmental period were significantly reduced over control when Azotobacter, PSB and vermicompost were used in different combinations with graded doses of RDF during both years of experimentation (Table 2). Significantly minimum fruit developmental period (140.25 and 141.37 days, respectively) was recorded when the plants were treated with RDF 75\% + Azotobacter $100 \mathrm{~g}+$ PSB $100 \mathrm{~g}+$ vermicompost $2 \mathrm{~kg} / \mathrm{plant}$, whereas the maximum fruit developmental period (164.00 and 163.26 days, respectively) was recorded in plants kept as untreated control. Application of graded dose of chemical fertilizers in association with organic manure and bio-fertilizers, may results in enhanced activity of biological nitrogen fixation and higher net assimilation rate on account of better growth leading to the production of endogenous metabolites. These results are in conformity with the findings of Srinu et al., (2017), Srivastava et al., (2014) in papaya.

\section{Number of flowers and fruits set per plant}

Data presented in Table 2, it is clearly reveals that during both years of experimentation number of flowers and fruits set per plant were significantly more over control when Azotobacter, PSB and vermicompost were used in combinations with different doses of RDF. Higher number of flowers (104.69 and 104.80 , respectively) with maximum number of fruits set (43.00 and 40.85, respectively) per plant was recorded in plants which were fertilized with RDF 75\%+ Azotobacter $100 \mathrm{~g}$ + PSB $100 \mathrm{~g}+$ vermicompost $2 \mathrm{~kg} / \mathrm{plant}$, whereas the minimum number of flowers (73.86 and 72.24, respectively) and fruits set (24.00 and 22.80, respectively) were recorded in plants which were kept under control without application of non-amount of fertilizers during both years of experimentation. This increase in number of flowers and fruits set in this treatment might be due to the fact that the application Azotobacter and PSB along with vermicompost and NPK fertilizers as a balanced dose accelerated the development of inflorescence, leaf number in autumn which results in increased levels of nutrients in assimilating area of crop due to which the rate of dry matter production was enhanced, which is positively correlated with the number of flowers and fruits in the following spring. Proper supply of nutrients and induction of growth hormones stimulated cell division, cell elongation, which results an increase in number of flowers and fruits. Similar findings have been reported by Srinu et al., (2017) in papaya, Gupta and Tripathi (2012) in strawberry cv. Chandler, Tripathi et al., (2015a) in aonla and Katiyar et al., (2012) in ber.

\section{Fruit yield}

Data presented in Table 1 clearly shows that significantly maximum fruit yield was recorded when plants were fertilized with Azotobacter, PSB and vermicompost in combinations with different doses of RDF during both years of experimentation. The maximum yield per plant (67.08 and 60.44 $\mathrm{kg}$, respectively) was recorded in plants fertilized with the combination of RDF 75\% + Azotobacter $100 \mathrm{~g}+$ PSB $100 \mathrm{~g}+$ vermicompost $2 \mathrm{~kg} /$ plant $\left(\mathrm{T}_{9}\right)$ and this yield was significantly higher as compared to all other treatments. Plants kept under control produced the minimum yield of fruits (22.34 and $23.49 \mathrm{~kg}$, respectively) during both years of experimentation. This increase in yield parameters during both years of experimentation with the use of vermicompost, Azotobacter and PSB in combination with graded dose of NPK might be due to an increased number of fruits set, higher nitrogen fixation in soil, increase in the 
availability of phosphorus with increased uptake of various other trace elements and translocation of photosynthates from leaves to developing fruits. Use of vermicompost results in increased microflora, which produce useful products in the soil, which ultimately helps in the biodegradation of various agricultural residues present in the soil. Relatively higher amount of carbohydrates could have promoted the growth rate and increased fruit weight. These findings are in line with the findings of Tripathi (2017), Nayyer et al., (2014) in banana, Tripathi et al., (2014), Tripathi et al., (2016) in strawberry, Kumar et al., (2015) in Guava, Srivastava et al., (2014), Srinu et al., (2017) and Kanwar et al., (2020) in papaya.

\section{Soil physical characters}

During both years of present investigation available potassium $\left(\mathrm{kg} \mathrm{ha}^{-1}\right)$ content in the soil have non-significant effect when Azotobacter, PSB and vermicompost were used in combinations with different doses of RDF during their production process (Table 3). However, maximum available potassium (270.14 and $272.65 \mathrm{~kg} \mathrm{ha}^{-1}$, respectively) content was recorded in the field fertilized with RDF 75\%+ Azotobacter $100 \mathrm{~g}+$ PSB $100 \mathrm{~g}+$ vermicompost $2 \mathrm{~kg} / \mathrm{plant}$, whereas the minimum available potassium (260.17 and $261.24 \mathrm{~kg} \mathrm{ha}^{-1}$, respectively) content was recorded in field kept under control during both years of experimentation. The reason for increased available $\mathrm{K}$ under inorganic and biofertilizer application may be due to the results of additional K supply, the solubilization action of certain organic acids produced during decomposition process and its greater capacity of hold $\mathrm{K}$ in available form in the soil and also due to the interaction of organic matters with clay and direct addition of potassium to the available pool of soil. Results are in conformity with the findings of Srivastava (2008), Yadav (2005) in guava.
Further investigation of orchard soil during both years of experimentation results that soil $\mathrm{pH}$, soil electrical conductivity, organic carbon per cent, available nitrogen and phosphorus contents in the soil were significantly influenced/increased over control, when Azotobacter, PSB and vermicompost were used in combinations with different doses of RDF during their production process (Table 3). During entire period of investigation minimum soil $\mathrm{pH}$ (7.10 and 7.26, respectively), soil electrical conductivity $\left(0.33\right.$ and $0.32 \mathrm{dSm}^{-1}$, respectively), maximum organic carbon per cent $(0.44$ and $0.45 \%$, respectively), maximum available nitrogen (271.15 and $300.85 \mathrm{~kg} \mathrm{ha}^{-1}$, respectively) and maximum available phosphorus (27.89 and $27.80 \mathrm{~kg}$ ha ${ }^{1}$, respectively) contents were recorded in the field fertilized with RDF $75 \%+$ Azotobacter $100 \mathrm{~g}+$ PSB $100 \mathrm{~g}+$ vermicompost $2 \mathrm{~kg} /$ plant, whereas the field having maximum soil $\mathrm{pH}$ (7.65 and 7.70, respectively), maximum electrical conductivity $\left(0.43\right.$ and $0.44 \mathrm{dSm}^{-1}$, respectively), minimum organic carbon per cent $(0.33$ and $0.34 \%$, respectively), minimum available nitrogen (251.26 and $252.34 \mathrm{~kg} \mathrm{ha}^{-1}$, respectively) and available phosphorus (20.35 and $21.95 \mathrm{~kg} \mathrm{ha}{ }^{-1}$, respectively) content were under control, where non amount of fertilizers were applied during both years of experimentation.

The significant reduction in $\mathrm{pH}$ and $\mathrm{EC}$ with the application of Azotobacter, PSB and vermicompost in combinations with different doses of inorganic fertilizers might be due to the fact that during decomposition of these manures and production of acidity with biofertilizers might have resulted in decline in soil $\mathrm{pH}$. The significant improvement in organic carbon content of soil with the application of Azotobacter, PSB and vermicompost in combinations with different graded doses of inorganic fertilizers may be 
due to the facts that Azotobacter, PSB and vermicompost results in death and decay of microorganism in the soil. Results for soil $\mathrm{pH}$, EC and organic carbon content are in close conformity with the findings of Babu et al., (2008) and Tiwari et al., (1999) in banana, Geo Jose et al., (2008).

The higher availability of nitrogen and phosphorus in the soil with the application of bio-fertilizers (Azotobacter and PSB) and vermicompost with inorganic fertilizers might be due to the reason that the Azotobacter not only fixes atmospheric nitrogen but also produces some organic acids, which stimulate the availability of other nutrients in the soil. PSB also induces some organic acids which help in solubilisation of unavailable phosphorus in soil. These results are in conformity with the findings of Gaurishankar et al., (2002), Krishnakumar et al., (2005), Chang et al., (2007), Reddy et al., (2010) in papaya.

\section{References}

Anonymous 2018. Horticultural statistics at a glance 2018, published by Government of India, Ministry of Agriculture \& Farmers' Welfare, Department of Agriculture, Cooperation \& Farmers' Welfare, Horticulture Statistics Division, New Delhi.

Babu R.P., Raghava Rao, D.V., Reddy, Y.N. and Madhava Rao. D. 2008. Organic banana production systems: Leaf emission, Leaf senescence and crop duration in plant and ratoon cycles. Indian Journal of Horticulture, 65(3):134-136.

Chang, E.H.; Chung, R.S. and Tsai, Y.H. 2007. Effect of different application rates of organic fertilizer on soil enzyme activity and microbial population. Soil Science and Plant Nutrition, 53:132-140
Gaurishankar; Verma, L.P. and Singh, Room 2002. Effect of integrated nutrient management on yield and quality of Indian mustard (B. juncea) and properties of soil. Indian Journal of Agricultural Sciences, 72 (9): 551-552.

Gautam, U. S.; Singh, R.; Tiwari, N.; Gurjar, P. S. and Kumar, A. 2012. Effect of integrated nutrient management in mango cv. Sunderaja. Indian Journal of Horticulture, 69 (2):151-155.

Geo Jose; Dhananjaya, B.C.; Prakash, H.C. and Sudhir, K. 2008. Labile fractions of soil organic matter as influenced by long term treatments in an alfisol (Typic rhodustalfs) under continuous rice pulse system. Mysore Journal of Agricultural Science, 42: 268-277.

Gupta, A.K. and Tripathi, V.K. 2012. Efficacy of Azotobacter and vermicompost alone and in combination on vegetative growth, flowering and yield of strawberry (Fragaria $x$ ananassa Duch.) cv. Chandler. Progressive Horticulture, 44 (2): 256261.

Jackson, M.L. 1973. Soil chemical analysis, prentice Hall of India, Pvt. Ltd, New Delhi.

Kanwar, A.; Sahu, G.D. and Panigrahi, H.K. 2020. Impact of integrated nutrient management on yield and quality parameters of papaya (Carica papaya L.) $\mathrm{Cv}$. Red Lady under net house. Journal of Pharmacognosy and Phytochemistry; 9(3): 1443-1445.

Katiyar, P.N., Tripathi, V.K., Sachan, R.K., Singh J. P. and Chandra, Ram 2012. Integrated Nutritional Management Effects on The Growth, Flowering, Fruiting and Yield of Rejuvenated Ber (Zizyphus mauritiana Lamk.) cv. Banarasi Karaka. Hort Flora Research Spectrum, 1 (1):38-41.

Kirankumar, R.; Jaganath, S.; Guruprasad, T.R.; Narayana, C. K.; Balakrishna5, A. 
N.; Venugopalan, R. and Anilkumar, S. 2017. Studies on Plant Density and Integrated Nutrient Management for Growth, Yield, Quality and Shelf Life of Guava cv. Lalit in Rainy Season. International Journal of Pure Applied Bioscience, 5 (2): 354-366

Krishnakumar, N.; Saravanan, A.; Natarajan, S.K.; Veerabadran, Y. and Mani, S. 2005. Microbial population and enzymatic activity as influenced by organic farming. Research Journal of Agricultural Biological Science, 1:8588

Kumar, A., Tripathi, V.K., Dubey, A.K. and Dubey, Vishal 2015. Effect of Integrated Nutrient Management on Fruits Set, Yield and Physico-Chemical Parameters of Winter Season Guava (Psidium guajava L.) cv. L-49. Progressive Research-An International Journal, 10 (Special-VII): 3722-37240.

Mamta, Dash, D.; Gupta, S.B. and Deole, Sonali. 2017. Effect of integrated nutrient management on growth and nutrient uptake in papaya (Carica papaya L.) at nursery level. Journal of Pharmacognosy and Phytochemistry, 6(5): 522-527.

Nayyer, M. A.; Tripathi, V. K.; Kumar, Sanjeev; Lal, Deepa and Tiwari, Bharat 2014. Influence of Integrated Nutrient Management on Growth, Yield and Quality of Tissue Cultured Banana (Musa $\times$ paradisiaca) cv. Grand Naine. Indian Journal of Agricultural Sciences, 84 (6): 680-683.

Olsen, S. R., Cole, C. V.; Watanabe, F. S. and Dean, L. A. 1954. Estimation of available phosphorus in soils by extraction with sodium bicarbonate. U. S. Department of Agriculture Circular No. 939. Banderis, A. D., D. H. Barter and K. Anderson. Agricultural and Advisor.

Reddy, Y.T.N.; Kurian, R. M.;
Ganeshamurthy, A.N. and Pannerselvam, P. 2010. Effect of organic nutrition practices on papaya (cv. Surya) fruit yield, quality and soil health. Journal of Horticulture Science, 5 (2): 124-127.

Srinu. B.; Manohar Rao.; Veena Joshi; Narender Reddy and Sharma, H. K. 2017. Effect of Different Integrated Nutrient Management on Growth, Yield and Quality of Papaya (Carica Papaya L.) cv. Red Lady. Bulletin of Environment, Pharmacology and Life Sciences, 6 (1): 132-135

Srivastava, A. 2008. Integrated nutrient management on papaya (Carica papaya L.). Ph.D. Thesis. NDUAT, Kumarganj, Faizabad (U.P.).

Srivastava, A.; Singh, J.K. and Singh, H.K. 2014. Integrated Nutrient Management on Growth and quality of Papaya (Carica papaya L.) cv. CO-7. The Asian Journal of Horticulture, 9 (2):390-395.

Subbiah, B.V. and Asija, G.L. 1956. A rapid procedure for the estimation of available nitrogen in soil, Current Science, 25: 258-260.

Sukhada, M.; Shivananda, T.N.; Iyengar, H.R.V. 1995. Uptake of 32P labeled superphosphate by endomycorrhizal papaya (Carica papaya) cv. Coorg Honey Dew. Journal Nuclear of Agriculture, 24(4):30-31.

Tiwari, D.K.; Hasan, M.A. and Chattopadhyay, P.K. 1999. Studies on the response of dwarf Cavendish banana (Musa AAA) to bio-fertilizer inoculation. Horticulture Journal, 14(1): 35-41.

Tripathi, V.K. 2017. Influence of Integrated Nutrient Management in Ratoon Crop of Tissue Cultured Banana. Progressive Research-An International Journal, 12 (Special-IV): 2577-2580.

Tripathi, V.K.; Bahadur, Shyam, Dubey, 
Vishal and Kumar, Abhinav 2015a. Influence of Integrated Nutrient Management on Yield and Physicochemical Parameters of Aonla cv. Na-7. Progressive Research-An International Journal,10 (Special-VI): 3493-3496.

Tripathi, V.K.; Kumar, Sanjeev and Gupta, A.K. 2015b. Influence of Azotobacter and vermicompost on growth, flowering, yield and quality of strawberry cv. Chandler. Indian Journal of Horticulture, 72(2): 201-205.

Tripathi, V.K.; Kumar, Sanjeev; Katiyar, P.N. and Nayyer, M. A. 2013. Integrated nutrient management in Isabgol (Plantago ovata Forsk.). Progressive Horticulture, 45(2): 302305.

Tripathi, V.K.; Kumar, Sanjeev; Kumar, Kaushal; Kumar, Sarvesh and Dubey,
Vishal 2016. Influence of Azotobacter, Azospirillum and PSB on vegetative growth, flowering, yield and quality of strawberry cv. Chandler. Progressive Horticulture, 48(1): 49-53.

Tripathi, V.K.; Mishra, A.N.; Kumar, Sanjeev and Tiwari, Bharat 2014. Efficacy of Azotobacter and PSB on Vegetative Growth, Flowering, Yield and Quality of Strawberry cv. Chandler. Progressive Horticulture, 46 (1): 48-53.

Walkley, A.J. and Black, I.A. 1934 Estimation of soil organic carbon by the chromic acid titration method. Soil Science, (37): 29-38.

Yadav, H.B. 2005. Studies of Integrated nutrient management in guava (Psidium guajava L.) cv. Sardar. Ph.D. Thesis, NDUA\&T, Kumarganj, Faizabad (U.P.).

\section{How to cite this article:}

Amar Singh and Tripathi, V. K. 2020. Influence of INM on Vegetative Growth, Fruiting, Yield and Soil Physical Characters in Papaya (Carica papaya L.). Int.J.Curr.Microbiol.App.Sci. 9(10): 3811-3822. doi: https://doi.org/10.20546/ijcmas.2020.910.438 